\title{
A Technical Study on the Design and Fabrication of a Broken Ceramics/Bottle Grinding Machine Powered by a 2 Stroke Spark ignition Engine.
}

\author{
Stephen Tambari ${ }^{1}$ Ibor Benjamin ${ }^{2}$, Kanee Sorbari ${ }^{3}$, Oruene Watson Daibi ${ }^{4}$, \\ Opuda Datonye ${ }^{5}$ \\ 1,2,3,4,5 Department of Mechanical Engineering, Rivers Stat Polytechnic, P.M.B 20Bori.
}

\begin{abstract}
In any human environment (Residential, industrial, commercial etc) wastes are often generated. These wastes (combustible wastes, organic wastes, metallic wastes, broken ceramics/bottles wastes) constitutes serious nuisance and pose health problems when not properly managed. Attempt to overcome this problem and control these waste, led to the development of broken ceramics/bottles grinding machine. In the design a two stroke spark ignition engine was incorporated. Littered broken bottles and ceramics were gathered from the environment and fed into the hopper where it was grinded into powdered form to be used as a replacement fir sharp sand in a sand blasting machine. The performance of the machine was evaluated the through its output capacity which was feted to be $45.58 \mathrm{~kg} / \mathrm{S}$ using a single stroke S.I engine
\end{abstract}

Keywords: grinding machine, ceramics/Bottles waste, design, Fabrication.

\section{Introduction}

This machine is designed to grind broken bottles and Ceramics plates to powder to be used:

- As replacement for sharp sand in a sand blasting operation. Which is called grit-blasting?

- As a technique for solid-waste management, aimed at eliminating broken bottles from the environment which can not be handled by traditional means of waste-management.

- As a raw material for producing new breakable Ceramic plates and glass-product.

\section{Description of machine parts}

The machine consists of the following essential components:

- 2 Stroke S.I Engine: To give drive required to cause elative motion on grinding disc.

- A threaded shaft: Is a square threaded at one-third of its stem, so that it can transfer materials pitch-wire to the grinding disc.

- A Mild Steel Hopper: To accommodate broken bottles and Ceramic plates prior to grinding

- Pulleys and Belts: To provide support, rigidity and alignment of the pulleys.

- Work-Table: To provide Support, rigidity and alignment of the pulleys.

\subsection{Purpose of Study:}

This study is aimed at showing the design, analysis, calculations and performance evaluation of broken Ceramic/Bottles grinding machine. It is also meant to unveil a substitute that can be used as replacement for sharp-sand used in sand blasting machines.

\subsection{Benefit of Study:}

The work serves as a technique for solid waste management since it depends on littered broken Ceramics/Bottles. The grinded particles achieved will be useful where there is lack of sharp sand for sand blasting operation. It will also help in recycling process.

\subsection{Engineering Principles:}

Bottles and Ceramics are extremely hard but brittle and cannot withstand shock or impact but breaks in the process. The grinding-plates are made of cast - steel alloy or alloyed-cast iron, which are hard and tough and thus an withstand shock loading without breaking. When both material are subjected to impact or compressive forces. The brittle material shatters and is grinded in the process. It is on this principle that the Ceramics/Bottles grinding machine is based. Ceramics/ Bottles grinding machine is based. Ceramic particles are subjected to compressive forces between two discs in relative motion and is ground in the process. 


\section{Materials and Method:}

The fabricated parts which include the Mild Steel hopper, the supporting frame, were welded and fastened together wring bolts and nuts. Other parts such as the pulleys, shafts were turned and bored wring centre lathe.

The power transmission from the motor was made possible through the following elements: The belt, pulleys, bearings and the shaft

\section{Design Consideration}

In the design of this machine, the following factors were considered. These includes:

- The strength of the materials for fabrication.

- Size and shape of the material to be grinded.

- Stainless materials to prevent corrosion.

\section{Design Datas from table}

\section{Design Analysis}

From the table of materials using plane carbon steel of $\mathrm{CO}$, where ultimate tensile strength is; $320-400 \mathrm{~N} / \mathrm{mm}^{2}$ and

Yield strength $(\delta \mathrm{y})$ is $=200 \mathrm{~N} / \mathrm{Mn}^{2}$

Then Shear Stress, $Z=(0.5$ to 0.577$) \delta y$.

And abo for shock factor and fatigue factor $\mathrm{K}_{\mathrm{b}}$ and $\mathrm{K}_{z}$ for a gradually applied load, $\mathrm{k}_{\mathrm{b}}=\mathrm{K}_{z}=1.0$ (Khurmi and Guta, Machine Design 2005)

So, $z=0.5 \delta y=0.5 \times 200$

$$
=100 \mathrm{~N} / \mathrm{mm}^{2}
$$

Yield Strength $(\delta y)=200 \mathrm{~N} / \mathrm{mm}^{2}$

Ultimate Strength $=320 \mathrm{~N} / \mathrm{mm}^{2}$

Therefore, Factor of safety;

F.O.S = Ultimate tensile strength Yield strength

=) F.O.S $=320=1.6$

$200=$

\section{Shaft Design}

Wp
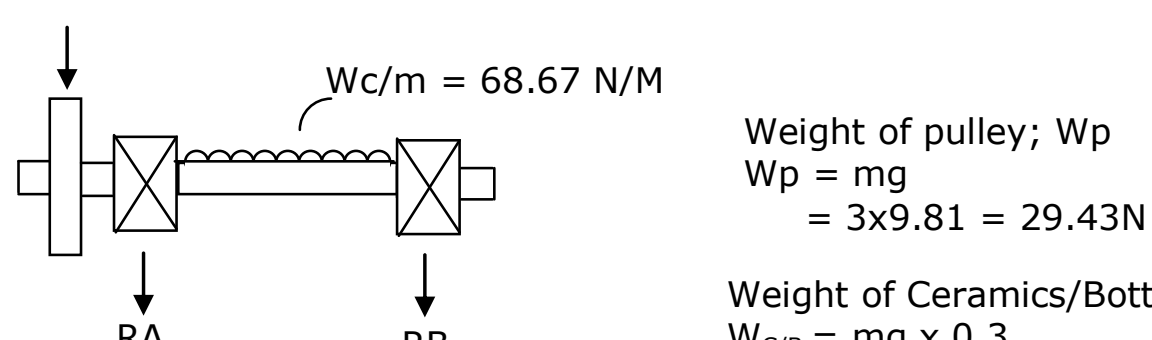

Weight of Ceramics/Bottles

RB

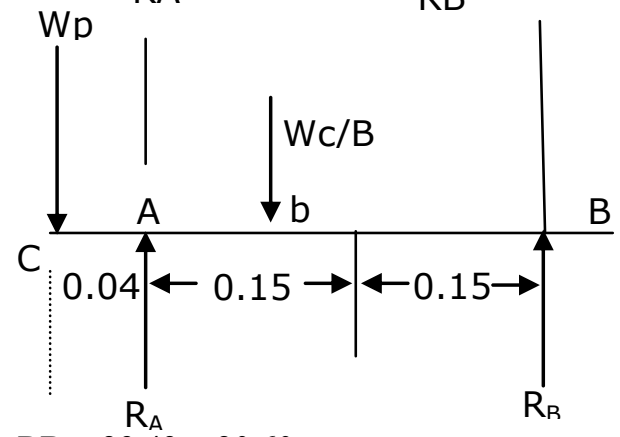

$\mathrm{W}_{\mathrm{C} / \mathrm{B}}=\mathrm{mg} \times 0.3$

$=7 \times 9.81 \times 0.3$

$=20.60 \mathrm{~N}$

$\mathrm{RA}+\mathrm{RB}=29.43+20.60$

$\mathrm{RA}+\mathrm{RB}=50.03 \mathrm{~N}$ 


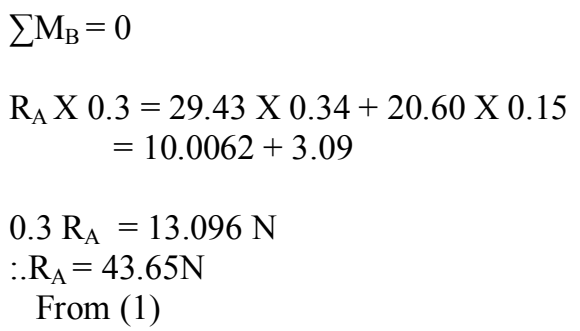

$R_{B}=50.03-63.65=6.38 \mathrm{~N}$

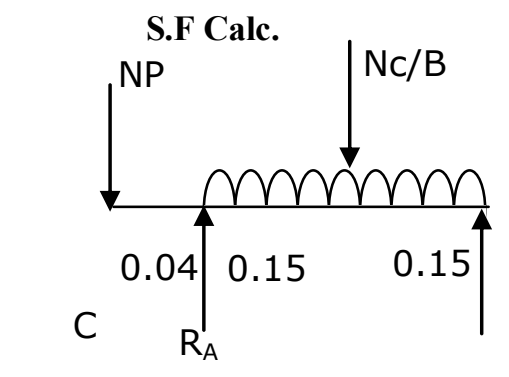

$$
\begin{aligned}
& \text { (a) P+.C: }: \mathrm{F}_{\mathrm{C}}=-29.43 \mathrm{~N} \\
& \text { (a) } \mathrm{P}+\text {. A: } \mathrm{S} \cdot \mathrm{F}_{\mathrm{A}}=-29.43+43.65 \\
& =+14.22 \mathrm{~N}
\end{aligned}
$$

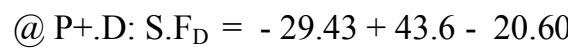

$$
\begin{aligned}
& \text { (a) } \mathrm{P}+\text {. B: } \mathrm{S} \cdot \mathrm{F}_{\mathrm{B}}=-6.38+6.38 \\
& =0 \text {. }
\end{aligned}
$$$$
=-6.38
$$$$
\mathrm{R}_{\mathrm{B}}
$$

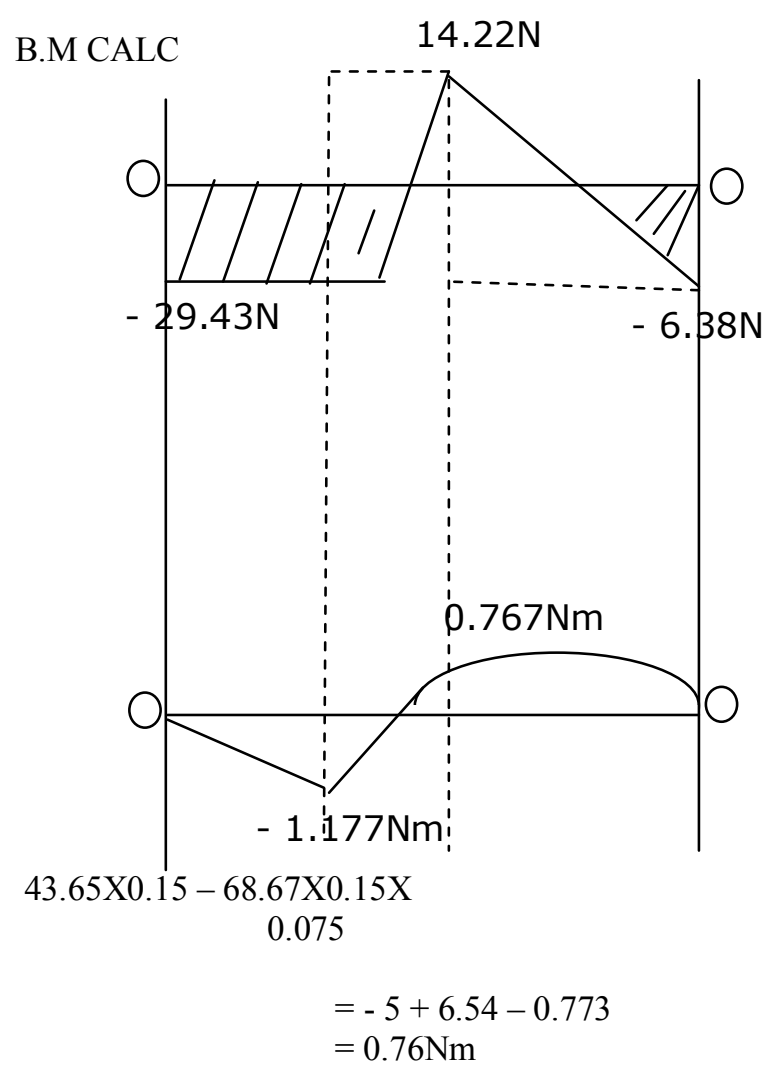

$$
\begin{aligned}
& \text { (a) C... } \mathrm{M}_{\mathrm{C}}=0 \\
& \begin{aligned}
\text { (a) P+. A: B.M } & =-29.43 \mathrm{X} 0.04 \\
& =1.177 \mathrm{NM}
\end{aligned}
\end{aligned}
$$

(a) P+ D: $\mathrm{B} \cdot \mathrm{M}_{\mathrm{D}}=-29.43 \times 0.19+$

The equivalent twist moment,

$$
\mathrm{Te}=\pi \times \mathrm{X} \mathrm{d}_{5}{ }^{3}
$$

Where: $Z=0.5 \delta y$ (Kharimi and Gupta machine design, 2005). 
$=) Z=0.5 \times 200$

$$
=100 \mathrm{~N} / \mathrm{mm}^{2}
$$

But Max. Bonding moment

$$
\begin{array}{cc}
\mathrm{Te}=0.767 \mathrm{Nm} & \text { where } \\
=767 \mathrm{Nmm} & \mathrm{d}_{\mathrm{s}}=\text { shaft diameter }
\end{array}
$$

$=) 767=\pi \times 100 \times \mathrm{d}_{\mathrm{s}}^{3}$

16

$=\mathrm{d}_{5}{ }^{3}=16 \times 767$

$$
3.142 \times 100
$$

$\mathrm{D}_{\mathrm{s}}{ }^{3}=\sqrt[3]{39.058}=19.00 \mathrm{~mm}$

$\therefore$ We choose $25 \mathrm{~mm}$ (standard size of shaft. See Khumi and Gupta machine design, 2005).

\section{Bearing selection analysis}

1. Subjecting bearing under the following condition.

2. Dynamic load on bearings

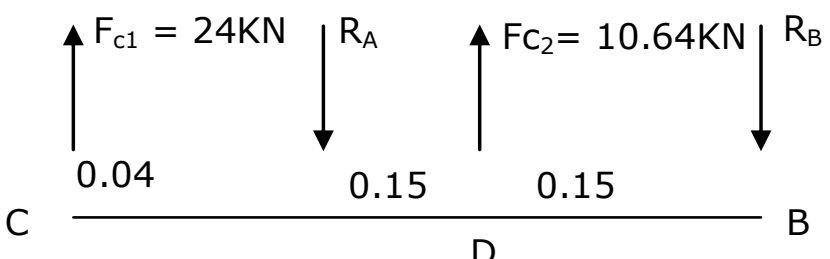

$\sum \mathrm{M}_{\mathrm{B}}=0$

$=) F_{c 1} \times 0.34+F_{c 2} \times 0.15=R_{A} \times 0.3$

$=) 0.3 \mathrm{R}_{\mathrm{A}}=24 \mathrm{X} 0.34+10.64 \times 0.15$

=) $0.3 R_{A}=8.16+1.596$

$$
\mathrm{R}_{\mathrm{A}}=9.756=32.52 \mathrm{KN}
$$

0.3

Also, $\mathrm{F}_{\mathrm{c} 1}+\mathrm{F}_{\mathrm{c} 2}=\mathrm{R}_{\mathrm{A}}+\mathrm{R}_{\mathrm{B}}$

${ }_{\Rightarrow} R_{B}=F_{c 1}+F_{c 2}-R_{A}$

$$
=24+10.64-32.52
$$

$\mathrm{R}_{\mathrm{B}}=2.12 \mathrm{KN}$

:. Dynamic loads are: $\mathrm{R}_{\mathrm{A}}=32.52 \mathrm{KN}$

$$
\mathrm{R}_{\mathrm{B}}=2.12 \mathrm{KN}
$$

Where: $\mathrm{F}_{\mathrm{c} 1}=\mathrm{mrw}^{2}$

$$
\begin{aligned}
& =7 * 0.0095 \\
& =* 400^{2} \\
& =10640 \mathrm{~N} \\
& =10.64 \mathrm{KN}
\end{aligned}
$$

$(\mathrm{M}=7, \mathrm{r}=$ shaft radius. Where $\mathrm{D}=19 \mathrm{~mm})$ 
$\mathrm{F}_{\mathrm{c} 2}=\mathrm{mrw}^{2}$

\begin{aligned}$\underline{F_{\mathrm{c} 2}} & =\mathrm{mrw}^{2} \\$\hline & $=3 \times 0.05 \times 400^{2} \\ & =24000 \mathrm{~N} \\ & =24 \mathrm{KN}\end{aligned}$

(Where $\mathrm{r}=$ pulley radius $\mathrm{D}=100 \mathrm{~mm}$ )

\section{(ii) STATIC LOADS}

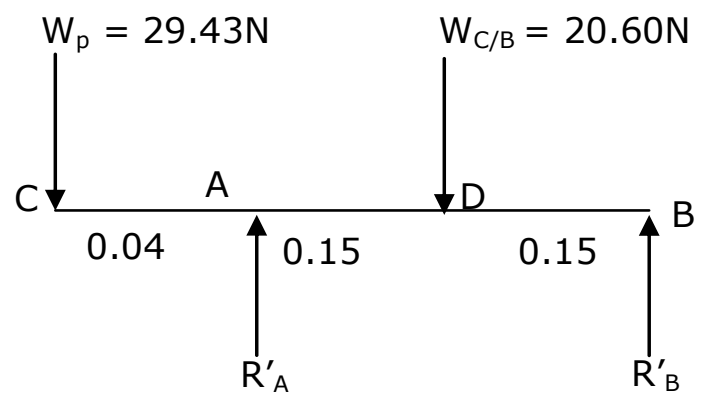

$\mathrm{R}_{\mathrm{A}}^{1}+\mathrm{R}_{\mathrm{B}}^{1}=29.43+20.60$

$\mathrm{R}_{\mathrm{A}}^{1}+\mathrm{R}_{\mathrm{B}}^{1}=50.03 \mathrm{~N}$

$\sum \mathrm{M}_{\mathrm{B}}=0$

$\mathrm{R}_{\mathrm{A}}^{1} \mathrm{X} 0.3=29.43 \times 0.34+20.60 \times 0.15$

$\therefore \mathrm{R}_{\mathrm{A}}^{1}=34.65 \mathrm{~N}=0.0437 \mathrm{KN}$

And $\mathrm{R}_{\mathrm{B}}^{1}=6.8 \mathrm{~N}=0.00638 \mathrm{KN}$

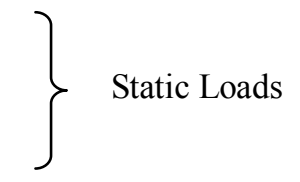

- The Maximum and Minimum loads on each bearing will be determine when the line of action of the dynamic loads coincide with the line of action of the static loads.

\section{THE SET UP NOW BECOMES:}

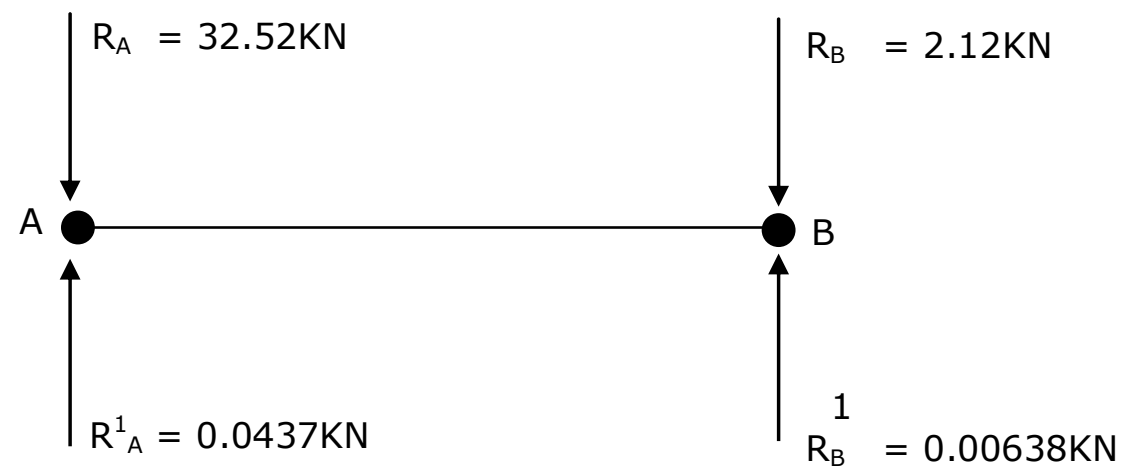

\section{Considering Bearing A:}

The maximum load on Bearing A:

$$
=\mathrm{R}_{\mathrm{A}}^{1}+\mathrm{R}_{\mathrm{A}}=32.52+0.0437
$$

$$
=32.564 \mathrm{KN}
$$

The maximum load on Bearing A: 


$$
\begin{gathered}
=\mathrm{R}_{\mathrm{A}}^{1}-\mathrm{R}_{\mathrm{A}}=32.52-0.0437 \\
=-32.476 \mathrm{KN}
\end{gathered}
$$

\section{Considering Bearing B:}

The maximum load on Bearing B:

$$
\begin{gathered}
=\mathrm{R}_{\mathrm{B}}^{1}+\mathrm{R}_{\mathrm{B}}=0.00638+2.12 \\
=2.126 \mathrm{KN}
\end{gathered}
$$

The minimum load on Bearing B:

$=\mathrm{R}_{\mathrm{B}}^{1}-\mathrm{R}_{\mathrm{A}}=0.00638-2.12$

$$
=-2.114 \mathrm{KN}
$$

Therefore, in the bearing selection process, the above load must be put into consideration.

\section{Design of the Hopper}

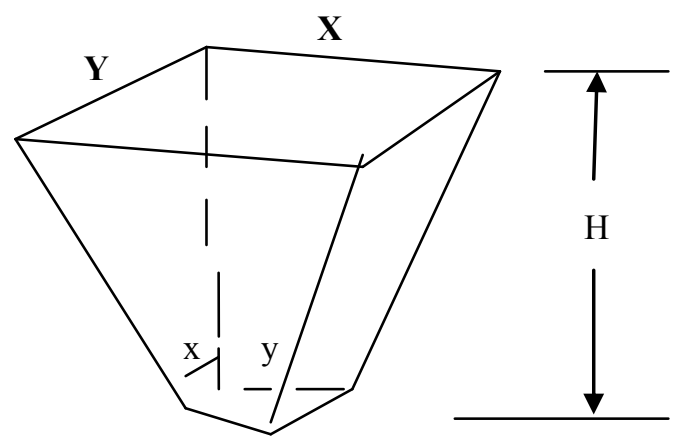

Length of upper rectangular dimension

$$
\begin{gathered}
X=\text { length }(\mathrm{L})=1225 \mathrm{~mm}=1.225 \mathrm{~m} \\
\mathrm{Y}=\text { width }(\mathrm{W})=735 \mathrm{~mm}=0.735 \mathrm{~m}
\end{gathered}
$$

The lower rectangular dimensions

$$
\begin{aligned}
& x=\text { length }(L)=122.5 \mathrm{~mm}=0.1225 \mathrm{~m} \\
& y=\text { width } \quad(W)=73.5 \mathrm{~mm}=0.0735 \mathrm{~m}
\end{aligned}
$$

The height of the hopper:

Height $=\mathrm{H}=490 \mathrm{~mm}=0.490 \mathrm{~m}$

Using the relationship:

Volume: $\left.\mathrm{V}=\frac{1}{3} \mathrm{H}\left[\left(X^{2} * Y-x^{2} * Y\right) /(X-x)\right)\right]$

$=\mathrm{V}=\frac{1}{3} \times 0.490 \frac{\left[\left(1.225^{2} * 0.735-0.1225^{2} * 0.0735\right)\right]}{(1.225-0.1225)}$

$\Rightarrow \mathrm{V}=0.1633\left[\left(\frac{1.10296-0.001103}{1.1025}\right)\right]$

$$
=0.1633 \times 0.999417
$$




$$
\begin{aligned}
& \mathrm{V}=0.1632 \mathrm{~m}^{3} \\
& \mathrm{~V}=163.2 \mathrm{~mm}^{3}
\end{aligned}
$$

\section{Belt Tensions}

The torque on pulley is same as torque on shaft.

$T_{A V}=\left(T_{1} T_{2}\right) r_{p}$ where $r_{p}=$ radius of pulley.

$\Rightarrow \mathrm{r}_{\mathrm{p}}=\frac{D 1}{2}$

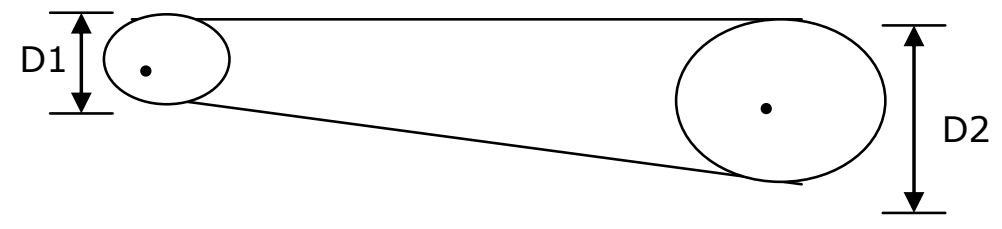

And $\mathrm{T}_{\mathrm{AV}}=$ average torque

$\mathrm{T}_{\mathrm{AV}}\left[T_{1}-T_{2}\right] \frac{D 1}{2}$

(1)

But $\frac{T_{1}}{T_{2}}=\mathrm{e}^{\mu_{\theta}}$

Where $\theta=(180-2 \phi)$ and groove angle $(\phi)$

Varies between $32^{0}$ to $40^{\circ}$ from table (Khurmi and Gupta, 2005)

So, using $\phi=32^{0}$

$\Rightarrow) \theta=\left[180-(2 \times 32]=180-64=116^{0}\right.$

And $\mu=$ fan $\phi=$ fan $32=0.62$

So, substituting values into (2)

$\therefore \frac{T_{1}}{T_{2}}=2.718^{0.62 \frac{116}{180}}$

$$
\begin{aligned}
& =2.718^{0.3996} \\
& =1.49
\end{aligned}
$$

$=) \frac{T_{1}}{T_{2}}=1.49$

(3)

Also,

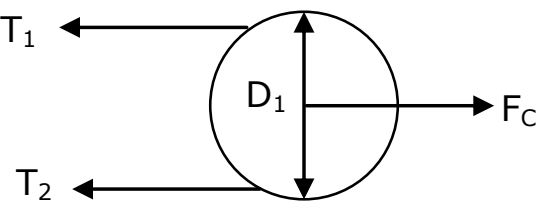

Using $\mathrm{D}_{1}=100 \mathrm{~mm}$ and

$\mathrm{D}_{2}=250 \mathrm{~mm}$

(Khuirmi and gupta 2005)

Where $\mathrm{D}_{1}=100 . \mathrm{mm}=0.1 \mathrm{~m}$ and $\mathrm{V}=20 \mathrm{~m} / \mathrm{s}$

(from table of datas. Khurmi and Gupta, 2005)

=) Angular velocity; $\mathrm{W}=\frac{V}{r}=\frac{20}{0.05}$ 
$=400 \mathrm{rad} / \mathrm{s}$

Centrifugal force, $\mathrm{F}_{\mathrm{C}}=\mathrm{mrw}^{2}$ [using $3.9 \mathrm{~kg}$ mars from table 1]

$\Rightarrow \mathrm{F}_{\mathrm{C}}=3.9 \times 0.05 \times 400^{2}$

$=31200 \mathrm{~N}=31.20 \mathrm{KN}$

$\sum \mathrm{M}_{\mathrm{T} 1}=0$

$\mathrm{T}_{2} \mathrm{X} 0.1=\mathrm{F}_{\mathrm{C}} \mathrm{X} 0.05$

$\therefore \mathrm{T}_{2}=\frac{31200 X 0.05}{0.1}$

$=15600 \mathrm{~N}=\underline{\underline{15.6 \mathrm{KN}}}$

SO, Putting $\mathrm{T}_{2}$ into eqn (1). We have;

$$
\begin{aligned}
\mathrm{T}_{1}=\mathrm{T}_{2} \times 1.49 & =15.6 \times 1.49 \\
& =\underline{\underline{23.24 \mathrm{KN}}}
\end{aligned}
$$

$\therefore$ Power $=\left(\mathrm{T}_{1-} \mathrm{T}_{2}\right) \mathrm{v}$

$$
\begin{aligned}
& =(23.24-15.6) 20 \\
& =.152 .88 \mathrm{~W}
\end{aligned}
$$

But $1 \mathrm{hp}=746$ Watts.

$\Rightarrow$ ) 152.88 Watts $=0.2 \mathrm{hp}$.

$\therefore$ The minimum power to drive the shaft is $(0.2 \mathrm{hp}-\mathrm{Ihp})$

Torque $\mathrm{T},=\left(\mathrm{T}_{1}=-\mathrm{T}_{2}\right) * \frac{D 1}{2}$

$\Rightarrow \mathrm{T}=(23.24-15.6) * \frac{0.1}{2}$

$$
=0.382 \mathrm{KNM}
$$

\section{The length of Belt}

$$
\mathrm{L}=2 \mathrm{C}+\frac{\pi}{2}\left(\mathrm{D}_{1}+\mathrm{D}_{2}\right)-\frac{D_{2}-D_{1}}{4 C}
$$

Where $\mathrm{C}=$ Centre distance, $\mathrm{m}$.

$$
\mathrm{C}=\left(\frac{D_{2}+D_{1}}{2}\right)+D_{1}
$$

So, using $\mathrm{D}_{1}=100 \mathrm{~mm}$ and $\mathrm{D}_{2}=250 \mathrm{~mm}$

(Khurim and Gupta, 2005)

$\Rightarrow C=\left(\frac{250+100}{2}\right)+100=275 \mathrm{~mm}$ 
$\therefore$ The length of Belt;

$$
\begin{aligned}
\mathrm{L} & =2 * 275+\frac{3.142}{2}(100+250)-\left(\frac{250-100}{4 * 275}\right) \\
\therefore \mathrm{L} & =550+549.85-0.1364 \\
& =1099.71 \mathrm{~mm}=1.099 \mathrm{~m}
\end{aligned}
$$

\section{Result}

The machine was test-run after fabrication in order to ascertain its performance. And the output capacity was also calculated. The result of the performance was summarized in table and table 2 below:

Table 1: Number ceramic/Bottles loading and the time taken to grind.

\begin{tabular}{|c|c|c|}
\hline Number of loading & Mass of Ceramic/Bottles (kg) & Time taken (Sec) \\
\hline 1 & 0.3 & 30 \\
\hline 2 & 1.0 & 60 \\
\hline 3 & 1.4 & 70 \\
\hline 4 & 1.8 & 120 \\
\hline 5 & 2.1 & 200 \\
\hline 6 & 2.5 & 230 \\
\hline 7 & 2.8 & 270 \\
\hline 8 & 3.2 & 300 \\
\hline 9 & 3.6 & 360 \\
\hline 10 & 3.9 & $\mathbf{1 7 8 5}$ \\
\hline
\end{tabular}

The output capacity of the machine

$$
=\frac{\text { TotalMass of Ceramics } / \text { Bottles }}{\text { timetaken }(\mathrm{hr})}=\frac{22.6 \times 3600}{1785}=45.58 \mathrm{~kg} / \mathrm{hr}
$$

Table 2: shows the relationship between particle size of grinded Ceramics and clearance between grinding plates.

\begin{tabular}{|c|c|c|}
\hline S/N & Disc Clearance (mm) & Grit sizes (mm) \\
\hline 1 & 1.0 & 0.8 \\
\hline 2 & 2.0 & 1.8 \\
\hline 3 & 3.0 & 2.7 \\
\hline 4 & 4.0 & 3.8 \\
\hline 5 & 5.0 & 4.8 \\
\hline 6 & 6.0 & 5.7 \\
\hline 7 & 7.0 & 6.8 \\
\hline 8 & 8.0 & 7.7 \\
\hline
\end{tabular}

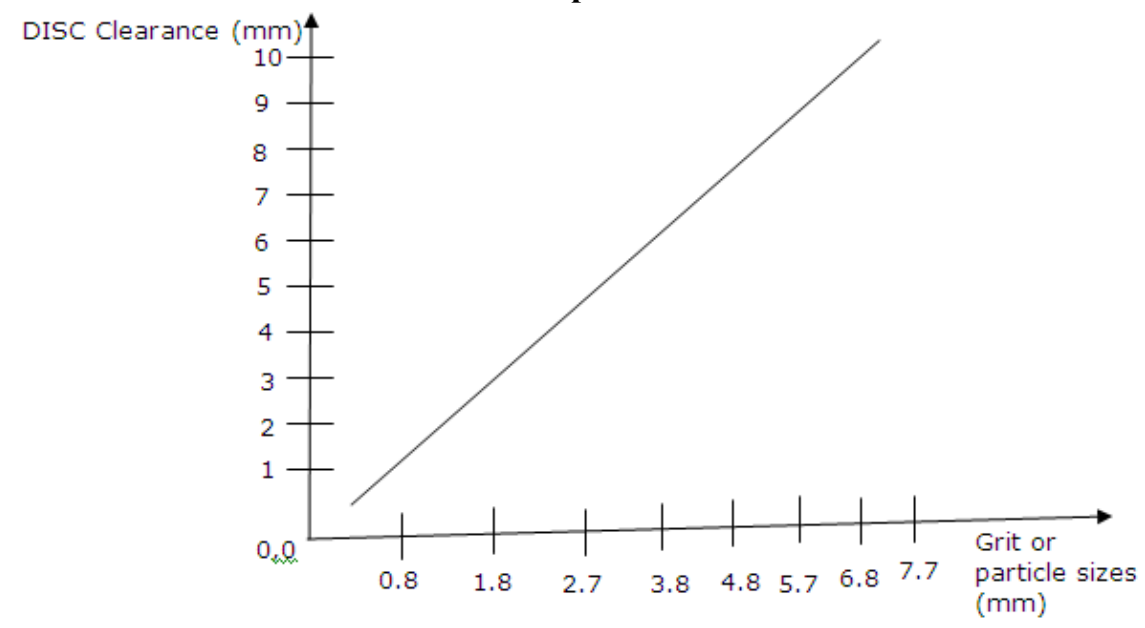



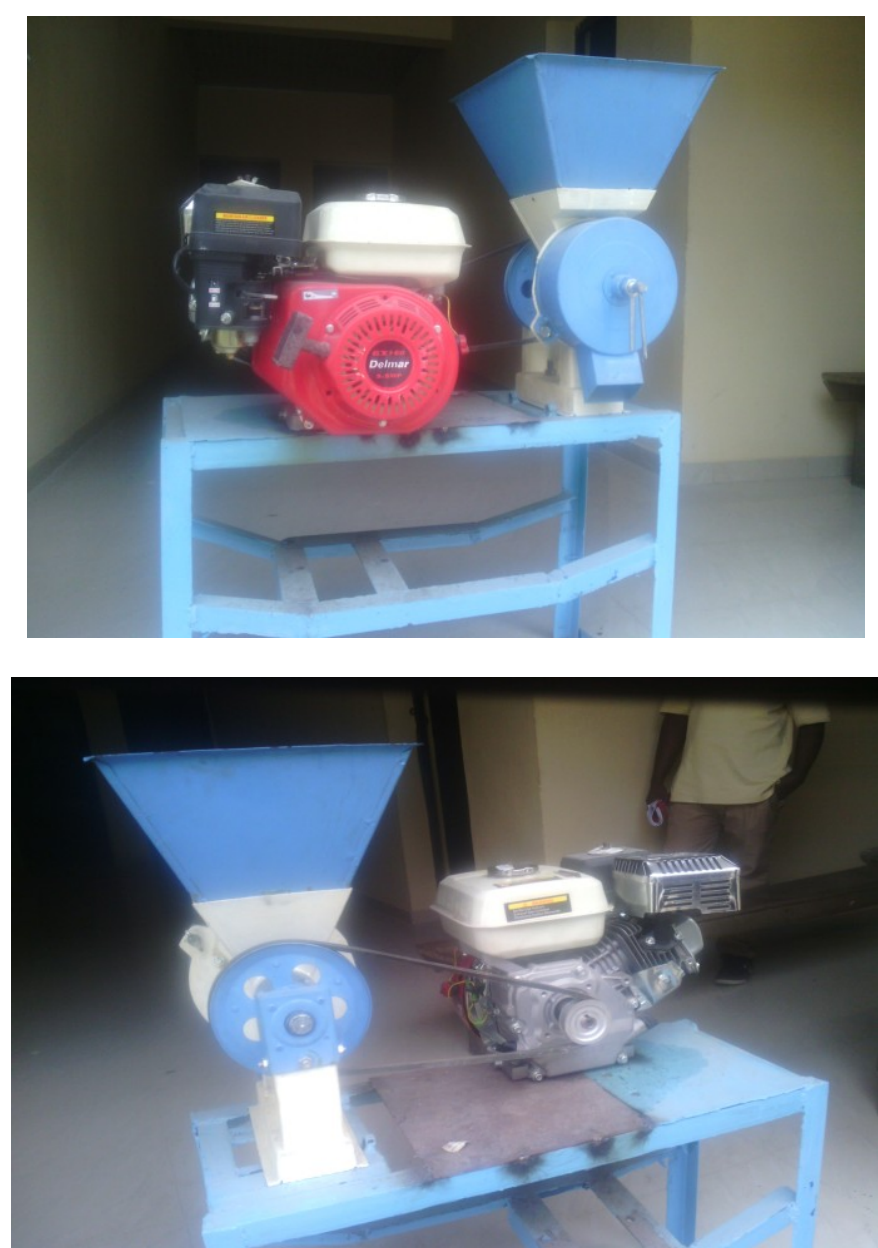
Flow chart 2:

Flow Chart 2. Showing The Full Procss

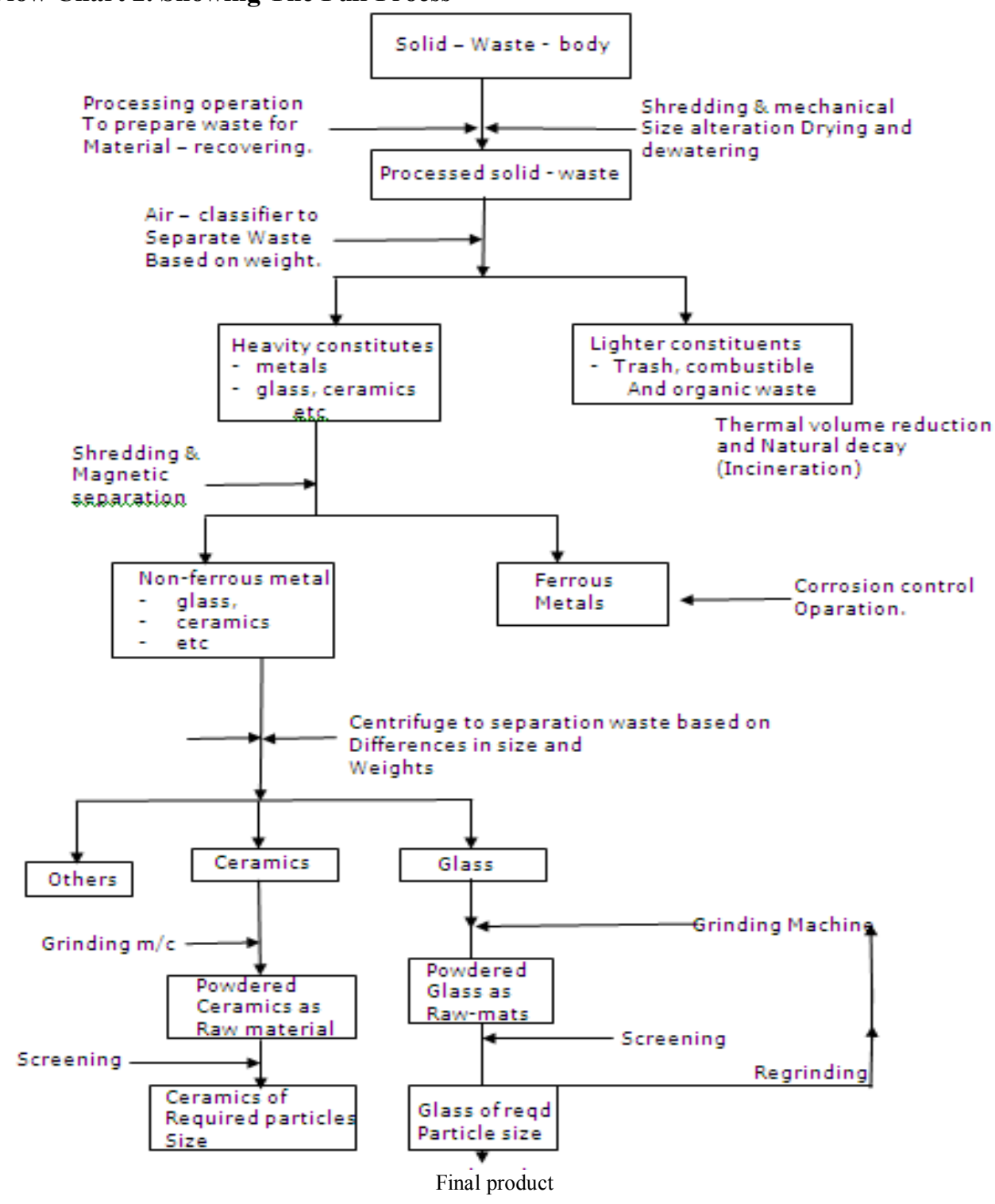

IV. Conclusion

A 2 shoke S.I engine: Power broken Ceramics/Bottles grinding machine was designed, Fabricated and tested. It was found to be effective and very efficient and could grind about $45.58 \mathrm{~kg}$ of Ceramics/Bottles per hour. This machine will help in the area of waste management and recycling of Ceramics and Bottles.

\section{Recommendation}

This machine can be used at any time since it does not depend on electricity for its operation. Is insufficient fuel to operate its, a humanly powered and a moterised powered machines is highly recommended as a replacement source of power for it to operate.

\section{References}

[1]. Samson E.A, Integrated Bicycle pedal, accessed on $24^{\text {th }}$ May, 2012 at Wikipe dia-free encyclopedia.

[2]. Ajao K.R, Mustaphak, Mahamood M.R, \& Lyanda M.O, (2010), Design and Development of a pedal - power Soap mixer, New York Science Journal, Vol.. 3(1) pP. 6 - 9

[3]. K.R Ajao, S.O Ayilara, I.O. usman, (2013), Design and Fabrication of a home Scale pedal power carsava Grater.

[4]. O.R. Adefunji, A.H. Quadri, (2011), Design and Fabrication of an Improved Cassava Grater. The practice journal of Science and Technology.

[5]. Hicks T.G, Standard Handbook of Engineering Calculations, $3^{\text {rd }}$ edition 1995.

[6]. Mrs. Uthman, F. (MNIAE), (2011), Design and Fabrication of cassava lump breaking and sieving machine.

[7]. Arthur, A (1974). Complete Junior Physics (5 ${ }^{\text {th }}$ ed), London: Longman Group limited.

[8]. Balogun, A.L (2006). "Design Fabrication and performance Evaluation of a Multi-Crop slicing Machine" An unpublished M. Eng. Thesis Submitted to the Department of Agricultural Engineering at the Federal University of Technology, Akure. 